\title{
Landau damping of dust acoustic solitary waves in nonthermal plasmas
}

\author{
Yashika Ghai a , N. S. Saini ${ }^{\mathrm{b}}$ \\ Department of Physics, Guru Nanak Dev University, Amritsar-143005, India \\ B. Eliasson ${ }^{\mathrm{c}}$ \\ SUPA, Department of Physics, University of Strathclyde, \\ Glasgow G4 ONG, Scotland, United Kingdom.
}

(Dated: December 20, 2017)

\begin{abstract}
Dust acoustic (DA) solitary and shock structures have been investigated under the influence of Landau damping in a dusty plasma containing two temperature nonthermal ions. Motivated by the observations of Geotail spacecraft that reported two-temperature ion population in the Earth's magnetosphere, we have investigated the effect of resonant wave-particle interactions on DA nonlinear structures. The KdV equation with an additional Landau damping term is derived and its analytical solution is presented. The solution has the form of a soliton whose amplitude decreases with time. Further, we have illustrated the influence of Landau damping and nonthermality of the ions on DA shock structures by a numerical solution of the Landau damping modified KdV equation. The study of the time evolution of shock waves suggests that an initial shock-like pulse forms an oscillatory shock at later times due to the balance of nonlinearity, dispersion and disspation due to Landau damping. The findings of the present investigation may be useful in understanding the properties of nonlinear structures in the presence of Landau damping in dusty plasmas containing two temperature ions obeying nonthermal distribution such as in the Earth's magnetotail.
\end{abstract}

\footnotetext{
${ }^{a}$ Email: yashu.gh92@gmail.com

b Email: nssaini@yahoo.com (Corresponding author)

${ }^{c}$ Email:bengt.eliasson@strath.ac.uk
} 


\section{INTRODUCTION}

Landau damping of collective oscillations in a collisionless plasma is caused by resonant wave-particle interactions ${ }^{1}$. Since the linear Landau damping of Langmuir waves was first predicted about five decades ago, Landau damping has now been identified in many different modes of collective oscillations in plasma. A nonlinear theory for ion acoustic waves modified by Landau damping due to electrons was proposed by Ott and Sudan ${ }^{2}$. They derived a KdV equation with Landau damping under the assumption that the ions are cold, i.e., $T_{i}=0$ and the particle trapping time is much larger than the Landau damping time, and investigated the dependence of steepening of waveform on the relative magnitudes of nonlinearity and Landau damping. Later, the Landau damping of ion acoustic wave in a Xenon plasma contaminated with Helium ions has been experimentally observed as a function of frequency in a uniform magnetic field ${ }^{3}$. A detailed theory pertaining to modification of the solitary solution due to resonant wave-particle interactions has been discussed. It has also been proposed that the resonant wave-particle interactions in a plasma may give rise to an acceleration of charged particles by transfer of wave energy to the charged species ${ }^{4}$. Thereafter, many researchers have reported theoretical and experimental studies of nonlinear Landau damping in different plasma systems ${ }^{5-11}$. An experiment to demonstrate the propagation of ion acoustic soliton in an Ar-He plasma has been reported and it is suggested that the addition of a Landau damping term in the KdV equation affects the balance of nonlinearity and dispersion which leads to modification of the evolutionary pattern of initial pulse ${ }^{12}$. This modification arises as the higher frequency components of the perturbation are assumed to suffer preferential damping. An experimental and numerical study has been reported to illustrate the influence of Landau damping of ion acoustic solitary waves and it has been observed that the magnitude of Landau damping due to ions is increased by increasing the temperature of ions ${ }^{13}$.

Micron, or sub-micron sized dust particles when suspended in a plasma can be electrically charged positively or negatively via processes such as thermionic emission, field emission, radioactivity, impact ionization, collection of background electrons and ions, etc ${ }^{14,15}$. The charged dust particles tend to significantly alter the dynamics of normal electron-ion plasma and form a dusty plasma ${ }^{16}$. Dusty plasmas have received a considerable attention in the past three decades due to their wide occurrence in space (cometary tails, asteroids zones, 
planetary rings, interstellar medium, Earth's ionosphere and magnetosphere, etc.) and laboratory environments. After the theoretical predictions of the low-frequency dust acoustic waves (DAWs) ${ }^{17}$ and the high-frequency dust ion acoustic waves (DIAWs) ${ }^{18}$, the novel physics associated with the propagation of waves in different dusty plasma environments has been investigated by many researchers around the world. The presence of negatively charged dust in the plasma leads to an electron depleted plasma. In the recent past, various investigations pertaining to dust acoustic nonlinear waves have been reported in electron depleted dusty plasma ${ }^{19,20}$. A nonlinear theory of dust acoustic solitary waves with Landau damping due to ions has been investigated in an unmagnetized electron depleted dusty plasma containing positive and negative ions ${ }^{5}$. It is found that for some typical plasmas in laboratory and space, the Landau damping and nonlinear effects are more dominant than the dispersive effects and hence, the KdV soliton theory ceases to govern the dynamics of dust acoustic waves in such plasma. Recently, the effects of Landau damping on low frequency solitary waves in a dusty plasma have been investigated taking into account the variation of dust charge ${ }^{6}$ and it is reported that the amplitude of DAW decreases with time due to the influence of Landau damping. However, all these investigations have been reported in context of solitary waves in plasmas.

It is assumed that the shock waves carry energy and momentum with them and may be responsible for accelerating elementary charged particles in our galaxy and thus give rise to $\operatorname{cosmic}$ rays $^{21}$. A delicate balance between nonlinearity and dissipation may give rise to the formation of shock waves in plasma where the dissipation may arise due to fluid viscosity, inter-particle collisions, non-adiabatic dust charge fluctuations or can be because of Landau damping. In the past, various particle heating mechanisms have been associated with shock waves, such as heating of cold ions in Earth's plasma sheet due to slow mode shocks ${ }^{22}$. A number of investigations have been reported for the study of shock waves in different types of plasmas using Burgers/KdV-Burgers equation ${ }^{19,20,23,24}$. Recently, analytical and experimental studies have been performed to investigate the effect of Landau damping of ion acoustic shock waves using the $\mathrm{KdV}$ equation with an additional Landau damping term ${ }^{7,8}$. But no investigation has been reported to study the propagation properties of dust acoustic shock waves undergoing Landau damping due to resonance of heavy ions with low frequency dust acoustic wave mode.

Satellite observations have reported the distributions of particles in plas- 
mas that significantly deviate from equilibrium distributions and are found to obey certain non-Maxwellian distributions such as Kappa-distribution or qnonextensive distribution. Recent investigations have considered the existence of different kinds of nonlinear structures in magnetized and unmagnetized dusty plasma containing electrons obeying q-nonextensive distribution. ${ }^{25,26}$. Another commonly employed distribution to study the distributions far removed from Maxwellian distributions is Cairns nonthermal distribution ${ }^{27,28}$ that has been successfully fitted to explain the density depletion in the upper ionosphere detected by Freja satellite ${ }^{29}$. Such a non-Maxwellian distribution has been employed to study nonlinear structures in different plasma environments ${ }^{23,30-33}$. The effect of Landau damping on a nonlinear ion-acoustic solitary wave in a magnetized plasma containing nonthermal ions has been reported by Bandyopadhyay and Das ${ }^{9}$. They derived an evolution equation, including the effects due to Landau damping and discussed its solitary solution. Further, the analysis was extended to derive a KdV-ZK equation to study a kinetic Alfvén and ion-acoustic waves in a nonthermal magnetized plasma containing warm ions ${ }^{10}$. Recent observations by the Geotail spacecraft have reported the presence of two-temperature (hot and a relatively cold) ion population in Earth's magnetotail ${ }^{34,35}$. Two energy peaks at $0.7 \mathrm{keV}$ and $7 \mathrm{keV}$ were observed where the cold ion population consisted mainly of $\mathrm{O}^{+}$ions, whereas the hot ions were mainly protons ( $H^{+}$ions) whose source is the solar wind. It is emphasized that due to their larger mass, the effect of inertia of the colder $\mathrm{O}^{+}$ions is not negligible when considering the dynamics of large scale phenomena in the Earth's magnetosphere ${ }^{34}$. The two ion populations having substantially higher temperatures can be assumed to follow a non-Maxwellian distribution such as Cairns distribution.

Motivated by the observations by the Geotail spacecraft in the magnetotail region of the Earth's magnetosphere, we have investigated the effect of Landau damping due to cold and heavier $\mathrm{O}^{+}$ions on DAWs propagating in the magnetotail ${ }^{34-37}$. The aim is to study the variation in characteristics of dust acoustic nonlinear structures (shocks and solitons) in the presence of Landau damping within a plasma containing two temperature nonthermal ions. The effect of Landau damping on the properties of dust acoustic waves in an electron depleted dusty plasma in presence of Cairns distributed two temperature ions has not been reported so far. We aim to highlight the effect of a nonthermal ion population on the propagation properties as well as Landau damping of DAWs. The results of this investigation may 
be useful to understand the resonant wave-particle interactions in dusty plasmas containing nonthermal ions as well as the formation of nonlinear structures in such plasma environments.

The manuscript is structured as follows. In Sec. II, the model equations for present investigation are presented and using the reductive perturbation method the KdV equation with Landau damping has been derived. In Sec. III, the analytical solution for Landau damping modified KdV-equation is derived. In Sec. IV, the numerical investigation and discussion is presented and Sec. V is devoted to concluding remarks of present investigation.

\section{THE FLUID EQUATIONS}

We consider an electron depleted dusty plasma consisting of a negatively charged inertial dust fluid and two temperature ions obeying a nonthermal Cairns distribution. The colder ion species is considered massive as compared to the hot ions and hence, we shall investigate Landau damping of DAWs due to cold heavier ions. We assume that the plasma is collisionless while neglecting the dissipation due to dust charge fluctuation. The fluid equations of motion are as follows:

$$
\begin{gathered}
\frac{\partial n_{d}}{\partial t}+\frac{\partial\left(n_{d} u_{d}\right)}{\partial x}=0, \\
\frac{\partial u_{d}}{\partial t}+u_{d} \frac{\partial u_{d}}{\partial x}=-\frac{q_{d}}{m_{d}} \frac{\partial \phi}{\partial x}, \\
\frac{\partial^{2} \phi}{\partial x^{2}}=-4 \pi e\left(n_{i 1}+n_{i 2}-Z_{d} n_{d}\right) .
\end{gathered}
$$

The dynamics of the ion species are governed by the Vlasov equation

$$
\frac{\partial f_{j}}{\partial t}+v \frac{\partial f_{j}}{\partial x}-\frac{q_{j}}{m_{j}} \frac{\partial \phi}{\partial x} \frac{\partial f_{j}}{\partial v}=0
$$

yielding the number density

$$
n_{j}=\int_{-\infty}^{\infty} f_{j} d v
$$

At equlibrium, the plasma is considered to be quasi-neutral and the charge neutrality condition is given by $n_{i 10}+n_{i 20}=Z_{d} n_{d 0}$ which yields

$$
\mu_{i 1}+\mu_{i 2}=1
$$

where, $\mu_{i 1}=\frac{n_{i 10}}{Z_{d} n_{d} 0}, \mu_{i 2}=\frac{n_{i 20}}{Z_{d} n_{d} 0}, Z_{d}$ is the charge and $n_{d}$ is the density of dust particles. We shall normalize the number densities of cold ions, hot ions and dust grains $n_{r}(r=i 1, i 2, d)$ by 
their unperturbed number densities $n_{r 0}(r=i 1, i 2, d)$. The speed and electrostatic potential shall be respectively normalized by $C_{d}=\left(\frac{Z_{d} K_{B} T_{i 1}}{m_{d}}\right)^{1 / 2}=\lambda_{D, d} \omega_{p, d}$ which is the dust acoustic speed and $\phi_{0}=\frac{K_{B} T_{i 1}}{e}$, where $\lambda_{D, d}=\left(\frac{K_{B} T_{i 1}}{4 \pi n_{d 0} Z_{d} e^{2}}\right)^{1 / 2}$ is the dust Debye length and $\omega_{p, d}^{-1}=$ $\left(\frac{m_{d}}{4 \pi n_{d 0} Z_{d}^{2} e^{2}}\right)^{1 / 2}$ is the inverse dust plasma frequency, $T_{i 1}$ is the temperature of the colder ion species, $m_{d}$ is the dust mass. $f_{j}$ is normalized by $\frac{n_{j 0}}{v_{t j}}$ and $v$ is normalized by $v_{t i 1}$, $v_{t j}=\sqrt{\frac{K_{B} T_{j}}{m_{j}}}$ is the thermal velocity of $j^{t h}$ ion species. Space and time shall be normalized by $L$ and $L / C_{d}$, respectively, where $L$ depicts the characteristic scale length for variation of physical quantities. The normalized equations can be written as

$$
\begin{gathered}
\frac{\partial n_{d}}{\partial t}+\frac{\partial\left(n_{d} u_{d}\right)}{\partial x}=0 \\
\frac{\partial u_{d}}{\partial t}+u_{d} \frac{\partial u_{d}}{\partial x}=\frac{\partial \phi}{\partial x} \\
\frac{\lambda_{d}^{2}}{L^{2}} \frac{\partial^{2} \phi}{\partial x^{2}}=-\mu_{i 1} n_{i 1}-\mu_{i 2} n_{i 2}+n_{d}
\end{gathered}
$$

and the normalized number densities as

$$
n_{j}=\sqrt{\frac{T_{i 1} m_{j}}{T_{j} m_{i 1}}} \int_{-\infty}^{\infty} f_{j} d v .
$$

The normalized Vlasov equation for ion species become

$$
\delta \frac{\partial f_{j}}{\partial t}+v \frac{\partial f_{j}}{\partial x}-\frac{m_{i 1}}{m_{j}} \frac{\partial \phi}{\partial x} \frac{\partial f_{j}}{\partial v}=0
$$

where $j=i 1, i 2$ for cold and hot ions, respectively.

- $\delta=\sqrt{\frac{Z_{d} m_{i 1}}{m_{d}}}$ represents the effects due to inertia of cold ions and in particular Landau damping by heavier ions.

- $\frac{n_{d 1}}{n_{d 0}}$ depicts the ratio of perturbed density to its equilibrium value which is a measure of the strength of nonlinearity in electrostatic disturbances.

- $\frac{\lambda_{D}{ }^{2}}{L^{2}}$ depicts the strength of the wave dispersion due to deviation from the charge quasi-neutrality, where $L$ represents the characteristic scale length for variations of the physical quantities, namely $n_{d}, u_{d}, \phi$ etc.

If the normalization of the space co-ordinate is performed by the dust Debye length, the term depicting the strength of the wave dispersion shall vanish in Eq.(8). One of our main 
interests is to study the interplay among the nonlinearity, the dispersion and the Landau damping effects. We consider ${ }^{2,5}$

$$
\begin{aligned}
\delta & =\gamma_{1} \epsilon, \\
\frac{n_{d 1}}{n_{d 0}} & =\gamma_{2} \epsilon, \\
\frac{\lambda_{D}^{2}}{L^{2}} & =\gamma_{3} \epsilon .
\end{aligned}
$$

where, $\gamma_{j}$, with $j=1,2,3$ is a constant of the order unity and $\epsilon$ is a smallness parameter. From Eq. (10), the Vlasov equations for the two ion species become

$$
\begin{gathered}
\gamma_{1} \epsilon \frac{\partial f_{i 1}}{\partial t}+v \frac{\partial f_{i 1}}{\partial x}-\frac{\partial \phi}{\partial x} \frac{\partial f_{i 1}}{\partial v}=0 \\
\gamma_{1} \epsilon \frac{\partial f_{i 2}}{\partial t}+v \frac{\partial f_{i 2}}{\partial x}-m \frac{\partial \phi}{\partial x} \frac{\partial f_{i 2}}{\partial v}=0
\end{gathered}
$$

where $m=\frac{m_{i 1}}{m_{i 2}}$. To study the evolution of nonlinear structures in a nonthermal dusty plasma under the effect of Landau damping, we will derive a characteristic equation using hybrid model equations (Eqs. (6)-(12)). Using the reductive perturbation method, we introduce the following stretched co-ordinates to derive the KdV equation with an additional Landau damping term:

$$
\xi=\epsilon^{1 / 2}(x-M t) \text { and } \tau=\epsilon^{3 / 2} t
$$

where $M$ is the nonlinear wave speed (relative to the frame) normalized by $C_{d}$. The dependent physical quantities are expanded in powers with respect to $\epsilon$ about equilibrium state as

$$
\begin{aligned}
n_{d} & =1+\gamma_{2} \epsilon n_{d}^{(1)}+\gamma_{2}^{2} \epsilon^{2} n_{d}^{(2)}+\ldots \\
u_{d} & =\gamma_{2} \epsilon u_{d}^{(1)}+\gamma_{2}{ }^{2} \epsilon^{2} u_{d}^{(2)}+\ldots \\
\phi & =\gamma_{2} \epsilon \phi^{(1)}+\gamma_{2}^{(2)} \epsilon^{2} \phi^{(2)}+\ldots \\
n_{j} & =1+\gamma_{2} \epsilon n_{j}^{(1)}+\gamma_{2}{ }^{2} \epsilon^{2} n_{j}^{(2)}+\ldots \\
f_{j} & =f_{j}^{(0)}+\gamma_{2} \epsilon f_{j}^{(1)}+\gamma_{2}^{(2)} \epsilon^{2} f_{j}^{(2)}+\ldots
\end{aligned}
$$

where $f_{j}{ }^{(0)}$ represents the equilibrium distribution function for ion species and $j=i 1, i 2$ for cold and hot ions. The cold and hot ions are assumed to obey the nonthermal Cairns distribution whose normalized distribution function has the form ${ }^{27,28}$ :

$$
f_{j}{ }^{(0)}=\frac{1}{\sqrt{2 \pi}\left(1+3 \alpha_{s}\right)}\left(1+\alpha_{s} v^{4}\left(\frac{T_{i 1} m_{j}}{T_{j} m_{i 1}}\right)^{2}\right) \exp \left(-v^{2} \frac{T_{i 1} m_{j}}{T_{j} m_{i 1}}\right)
$$


where, $\alpha_{s}=\alpha_{1}, \alpha_{2}$ is the nonthermal parameter for cold and hot ions respectively. Substituting Eqs. (13)-(14) in Eqs. (6)-(9) and Eqs. (11)-(12), then equating the coefficients multiplying $\epsilon$ and $\epsilon^{2}$ to zero, we obtain the equations for the first and second order quantities.

\section{A. First-order perturbations and nonlinear wave speed}

At lowest order of $\epsilon$, equating the coefficients of $\epsilon^{3 / 2}$ from Eqs.(6)-(9) and Eqs. (11)-(12) to zero, we get the first order equations

$$
\begin{gathered}
-M \frac{\partial n_{d}^{(1)}}{\partial \xi}+\frac{\partial u_{d}^{(1)}}{\partial \xi}=0 \\
-M \frac{\partial u_{d}^{(1)}}{\partial \xi}+\frac{\partial \phi^{(1)}}{\partial \xi}=0 \\
-\mu_{i 1} n_{i 1}^{(1)}-\mu_{i 2} n_{i 2}^{(1)}+n_{d}^{(1)}=0, \\
n_{j}^{(1)}=\sqrt{\frac{T_{i 1} m_{j}}{T_{j} m_{i 1}}} \int_{-\infty}^{\infty} f_{j}^{(1)} d v \\
v \frac{\partial f_{i 1}^{(1)}}{\partial \xi}-\frac{\partial \phi^{(1)}}{\partial \xi} \frac{\partial f_{i 1}^{(0)}}{\partial v}=0 \\
v \frac{\partial f_{i 2}^{(1)}}{\partial \xi}-m \frac{\partial \phi}{\partial \xi} \frac{\partial f_{i 2}^{(0)}}{\partial v}=0
\end{gathered}
$$

from Eqs. (16)-(17), we obtain

$$
\begin{aligned}
& n_{d}^{(1)}=\frac{-\phi^{(1)}}{M^{2}}, \\
& u_{d}^{(1)}=\frac{-\phi^{(1)}}{M} .
\end{aligned}
$$

Eq.(20) yields

$$
\frac{\partial f_{i 1}^{(1)}}{\partial \xi}=-f_{i 1}^{(0)} \frac{\partial \phi^{(1)}}{\partial \xi}+\lambda(\xi, \tau) \delta(v),
$$

where $\delta(v)$ is Dirac's delta function and $\lambda(\xi, \tau)$ is an arbitrary function of $\xi$ and $\tau$. The above solution for $\frac{\partial f_{i 1}^{(1)}}{\partial \xi}$ involves the arbitrary function $\lambda(\xi, \tau)$, and hence is not unique. Thus, for a unique solution to exist, the problem should be posed as an initial value problem. Following Ott and Sudan ${ }^{2}$, we include a term $\epsilon^{7 / 2} \gamma_{1} \frac{\partial f_{i 1}^{(1)}}{\partial \tau}$ originating from the third order of $\epsilon$ in Eq.(11) after the expressions (13) and (14) being substituted. Thus, we write Eq.(20) as

$$
\gamma_{1} \epsilon^{2} \frac{\partial f_{i 1 \epsilon}^{(1)}}{\partial \tau}+v \frac{\partial f_{i 1 \epsilon}^{(1)}}{\partial \xi}-\frac{\partial \phi^{(1)}}{\partial \xi} \frac{\partial f_{i 1 \epsilon}^{(1)}}{\partial v}=0
$$


Similarly, Eq. (21) becomes

$$
\gamma_{1} \epsilon^{2} \frac{\partial f_{i 2 \epsilon}^{(1)}}{\partial \tau}+v \frac{\partial f_{i 2 \epsilon}^{(1)}}{\partial \xi}-m \frac{\partial \phi^{(1)}}{\partial \xi} \frac{\partial f_{i 2 \epsilon}^{(1)}}{\partial v}=0
$$

The solutions of the initial value problems (25) and (26) can now be found uniquely, once $f_{j \epsilon}^{(1)}$ for $j=i 1, i 2$ are known, by letting $\epsilon \rightarrow 0$ as

$$
f_{j(1)}=\lim _{\epsilon \rightarrow 0} f_{j \epsilon}{ }^{(1)}
$$

Next, taking the Fourier transform of Eq. (25) with respect to $\xi$ and $\tau$,

$$
\hat{f}(\omega, k)=\int_{-\infty}^{\infty} \int_{-\infty}^{\infty} f(\xi, \tau) \exp \iota(k \xi-\omega \tau) d \xi d \tau
$$

we obtain

$$
\hat{f}_{i 1 \epsilon}^{(1)}=-\frac{k \frac{\partial f_{i 1}^{(0)}}{\partial v}}{k v-\omega \gamma_{1} \epsilon^{2}} \hat{\phi}_{1} .
$$

To avoid the singularity appearing in Eq.(29), we replace $\omega$ by $\omega+\iota \eta$, where $\eta(>0)$ is a small parameter, to obtain

$$
{\hat{f_{i 1 \epsilon}}}^{(1)}=-\frac{k \frac{\partial f_{i 1}^{(0)}}{\partial v}}{k v-\omega \gamma_{1} \epsilon^{2}-\iota \eta \gamma_{1} \epsilon^{2}} \hat{\phi}_{1}
$$

Proceeding to the limit $\epsilon \rightarrow 0$ and using the Plemelj's formula

$$
\lim _{\epsilon \rightarrow 0} \frac{1}{k v-\omega \gamma_{1} \epsilon^{2}}=P\left(\frac{1}{k v}\right)+\iota \pi \delta(k v)
$$

where $P$ and $\delta$ denote the Cauchy principal value and Dirac's delta function, respectively, we obtain

$$
\hat{f}_{i 1}^{(1)}=2 \frac{\partial f_{i 1}^{(0)}}{\partial v^{2}} \hat{\phi}_{1}
$$

where we have used the properties $x P\left(\frac{1}{x}\right)=1, x \delta(x)=0$. Next, taking the inverse Fourier transform of the Eq. (32), we obtain

$$
f_{i 1}^{(1)}=2 \frac{\partial f_{i 1}^{(0)}}{\partial v^{2}} \phi_{1}
$$

Proceeding in the same way as above for the hotter ions, we obtain from Eq.(26)

$$
f_{i 2}^{(1)}=2 m \frac{\partial f_{i 2}^{(0)}}{\partial v^{2}} \phi_{1}
$$


From Eqs. (33) and (34) and using Eq. (19), we obtain the number density for the cold and hot ion species as

$$
\begin{aligned}
& n_{i 1}^{(1)}=-C_{i 1}^{(1)} \phi_{1} \\
& n_{i 2}^{(1)}=-C_{i 2}{ }^{(1)} \phi_{1}
\end{aligned}
$$

where $C_{i 1}{ }^{(1)}=\left(1-\beta_{i 1}\right)$ and $C_{i 2}{ }^{(1)}=\sigma\left(1-\beta_{i 2}\right)$, with $\beta_{i 1}=\frac{4 \alpha_{1}}{1+3 \alpha_{1}}, \beta_{i 2}=\frac{4 \alpha_{2}}{1+3 \alpha_{2}}$ and $\sigma=\frac{T_{i 1}}{T_{i 2}}$. Substituting the expressions for $n_{j}^{(1)}$ from Eqs.(35) and (36) in Eq. (18), we obtain

$$
M=\frac{1}{\sqrt{\left(\mu_{i 1} C_{i 1}{ }^{(1)}+\mu_{i 2} C_{i 2}{ }^{(1)}\right)}},
$$

which is the expression for nonlinear wave speed.

\section{B. Second-order perturbations}

At higher order of $\epsilon$, equating coefficients of $\epsilon^{(5 / 2)}$ from Eqs. (6) and (7), the coefficients

of $\epsilon^{2}$ from Eqs. (8) and (9) and the coefficients of $\epsilon^{(5 / 2)}$ from Eqs. (11) and (12) to zero, we successively obtain

$$
\begin{aligned}
& \frac{\partial n_{d}^{(1)}}{\partial \tau}-M \frac{\partial n_{d}^{(2)}}{\partial \xi}+\frac{\partial u_{d}^{(2)}}{\partial \xi}+\frac{\partial\left(n_{d}^{(1)} u_{d}^{(1)}\right)}{\partial \xi}=0 \\
& \frac{\partial u_{d}^{(1)}}{\partial \tau}-M \frac{\partial u_{d}^{(2)}}{\partial \xi}+u_{d}^{(1)} \frac{\partial u_{d}^{(1)}}{\partial \xi}-\frac{\partial \phi^{(2)}}{\partial \xi}=0 \\
& \frac{\lambda_{d}^{2}}{L^{2}} \frac{\partial^{2} \phi^{(1)}}{\partial \xi^{2}}=-\mu_{i 1} n_{i 1}^{(2)}-\mu_{i 2} n_{i 2}^{(2)}+n_{d}^{(2)} \\
& n_{j}^{(2)}=\sqrt{\frac{m_{j} T_{i 1}}{m_{i 1} T_{j}}} \int_{-\infty}^{\infty} f_{j}^{(2)} d v \\
& v \frac{\partial f_{i 1}^{(2)}}{\partial \xi}-\frac{\partial \phi^{(2)}}{\partial \xi} \frac{\partial f_{i 1}^{(0)}}{\partial v}=\gamma_{1} M \frac{\partial f_{i 1}^{(1)}}{\partial \xi}+\frac{\partial \phi^{(1)}}{\partial \xi} \frac{\partial f_{i 1}^{(1)}}{\partial v} \\
& v \frac{\partial f_{i 2}^{(2)}}{\partial \xi}-m \frac{\partial \phi^{(2)}}{\partial \xi} \frac{\partial f_{i 2}^{(0)}}{\partial v}=\gamma_{1} M \frac{\partial f_{i 2}^{(1)}}{\partial \xi}+m \frac{\partial \phi^{(1)}}{\partial \xi} \frac{\partial f_{i 2}^{(1)}}{\partial v}
\end{aligned}
$$

Substituting the expression for $f_{j}^{(1)}$ from Eqs.(33) and (34) into Eqs. (42) and (43), we successively obtain

$$
\begin{aligned}
& v \frac{\partial f_{i 1}^{(2)}}{\partial \xi}-\frac{\partial \phi^{(2)}}{\partial \xi} \frac{\partial f_{i 1}^{(0)}}{\partial v}=2 C_{a 1} \frac{\partial f_{i 1}^{(0)}}{\partial v^{2}}+4 v D_{a 1} \frac{\partial^{2} f_{i 1}^{(0)}}{\partial\left(v^{2}\right)^{2}} \\
& v \frac{\partial f_{i 2}^{(2)}}{\partial \xi}-m \frac{\partial \phi^{(2)}}{\partial \xi} \frac{\partial f_{i 2}^{(0)}}{\partial v}=2 C_{a 2} \frac{\partial f_{i 2}^{(0)}}{\partial v^{2}}+4 v m^{2} D_{a 2} \frac{\partial^{2} f_{i 2}^{(0)}}{\partial\left(v^{2}\right)^{2}}
\end{aligned}
$$


where, $C_{a 1}=\gamma_{1} M \frac{\partial \phi^{(1)}}{\partial \xi}, D_{a 1}=\phi_{1} \frac{\partial \phi^{(1)}}{\partial \xi} ; C_{a 2}=\gamma_{1} M \sigma \frac{\partial \phi^{(1)}}{\partial \xi}$ and $D_{a 2}=\sigma^{2} \phi_{1} \frac{\partial \phi^{(1)}}{\partial \xi}$.

Proceeding as above, to obtain unique solutions for $f_{j}{ }^{(2)}$ for cold $(j=i 1)$ and hot ions $(j=i 2)$, we introduce an extra higher order term $\epsilon^{9 / 2} \gamma_{1}\left(\frac{\partial f_{j}^{(1)}}{\partial \tau}\right)$ originating from the term $\epsilon^{5 / 2} \gamma_{1}\left(\frac{\partial f_{j}}{\partial \tau}\right)$ in Eqs.(11) and (12) after substituting Eqs. (13) and (14). Thus, Eqs. (44) and (45) are rewritten as

$$
\begin{aligned}
& \gamma_{1} \epsilon^{2} \frac{\partial f_{i 1}^{(2)}}{\partial \tau}+v \frac{\partial f_{i 1}^{(2)}}{\partial \xi}-\frac{\partial \phi^{(2)}}{\partial \xi} \frac{\partial f_{i 1}^{(0)}}{\partial v}=2 C_{a 1} \frac{\partial f_{i 1}^{(0)}}{\partial v^{2}}+4 v D_{a 1} \frac{\partial^{2} f_{i 1}^{(0)}}{\partial\left(v^{2}\right)^{2}} \\
& \gamma_{1} \epsilon^{2} \frac{\partial f_{i 2}^{(2)}}{\partial \tau}+v \frac{\partial f_{i 2}^{(2)}}{\partial \xi}-m \frac{\partial \phi^{(2)}}{\partial \xi} \frac{\partial f_{i 2}^{(0)}}{\partial v}=2 C_{a 2} \frac{\partial f_{i 2}^{(0)}}{\partial v^{2}}+4 v m^{2} D_{a 2} \frac{\partial^{2} f_{i 1}^{(0)}}{\partial\left(v^{2}\right)^{2}}
\end{aligned}
$$

In order to obtain unique solutions, we need to find $f_{j}^{(2)}$ for both ion species by letting $\epsilon \rightarrow 0$,

$$
f_{j}^{(2)}=\lim _{\epsilon \rightarrow 0} f_{j \epsilon}{ }^{(2)}
$$

Taking Fourier transform of Eq. (46), we get

$$
\hat{f}_{i 1}^{(2)}=\frac{k \frac{\partial f_{i 1}(0)}{\partial v} \hat{\phi}^{(2)}}{k v-\omega \gamma_{1} \epsilon^{2}}-2 \iota \frac{\hat{C}_{a 1} \frac{\partial f_{i 1}(0)}{\partial v^{2}}}{k v-\omega \gamma_{1} \epsilon^{2}}-\frac{\iota 4 v \hat{D}_{a 1} \frac{\partial^{2} f_{i 1}(0)}{\partial\left(v^{2}\right)^{2}}}{k v-\omega \gamma_{1} \epsilon^{2}} .
$$

In order to avoid singularity, $\omega$ is assumed to have a small positive imaginary part. So, we replace $\omega$ by $\omega+\iota \eta$, where $\eta>0$ and obtain from Eq. (49) as

$$
\hat{f}_{i 1}^{(2)}=\frac{k \frac{\partial f_{i 1}(0)}{\partial v} \hat{\phi}^{(2)}}{k v-\omega \gamma_{1} \epsilon^{2}-\iota \eta \gamma_{1} \epsilon^{2}}-2 \iota \frac{\hat{C}_{a 1} \frac{\partial f_{i 1}(0)}{\partial v^{2}}+2 v \hat{D_{a 1}} \frac{\partial^{2} f_{i 1}(0)}{\partial\left(v^{2}\right)^{2}}}{k v-\omega \gamma_{1} \epsilon^{2}-\iota \eta \gamma_{1} \epsilon^{2}} .
$$

Proceeding to the limit $\epsilon \rightarrow 0$ similar to the case of first order perturbed equations, using Plemlj's formula from Eq. (31) and using $x P(1 / x)=1, x \delta(x)=0$ and $\delta(k v)=\frac{\delta(v)}{|k|}$, we obtain the expressions for the number densities of the cold and hot ion species as

$$
\begin{aligned}
& \frac{\partial n_{i 1}{ }^{(2)}}{\partial \xi}+a_{1} \frac{\partial \phi}{\partial \xi}-a_{2} P \int_{-\infty}^{\infty} \frac{\partial \phi^{(1)}}{\partial \xi^{\prime}} \frac{\partial \xi^{\prime}}{\left(\xi-\xi^{\prime}\right)}-a_{3} \phi^{(1)} \frac{\partial \phi^{(1)}}{\partial \xi}=0 \\
& \frac{\partial n_{i 2}(2)}{\partial \xi}+b_{1} \frac{\partial \phi}{\partial \xi}-b_{2} P \int_{-\infty}^{\infty} \frac{\partial \phi^{(1)}}{\partial \xi^{\prime}} \frac{\partial \xi^{\prime}}{\left(\xi-\xi^{\prime}\right)}-b_{3} \phi^{(1)} \frac{\partial \phi^{(1)}}{\partial \xi}=0
\end{aligned}
$$

where the coefficients for cold ion populations are

$$
a_{1}=1-\beta_{i 1}, a_{2}=M \gamma_{1} \frac{\left(4-3 \beta_{i 1}\right)}{4 \sqrt{2 \pi}}, a_{3}=1 .
$$

For the hot ions population, the coefficients become

$$
b_{1}=\sigma\left(1-\beta_{i 2}\right), b_{2}=M \gamma_{1} \frac{\sigma^{3 / 2} \sqrt{m}\left(4-3 \beta_{i 2}\right)}{4 \sqrt{2 \pi}}, b_{3}=\sigma^{2}
$$


In order to obtain the required KdV-equation, we first eliminate $\partial u_{d}^{(2)} / \partial \xi$ and $\partial n_{d}{ }^{(2)} / \partial \xi$ from Eqs. (38)-(40) and then eliminate $\partial n_{j}{ }^{(2)} / \partial \xi$ by using Eqs. (51) and (52). In the resulting equation, we also substitute the expressions for $n_{d}^{(1)}$ and $u_{d}^{(1)}$ and obtain the following equation where the constants $\gamma_{1}, \gamma_{2}$ and $\gamma_{3}$ will enter into the Landau damping, nonlinear and the dispersive terms:

$$
\frac{\partial n}{\partial \tau}+A P \int_{-\infty}^{\infty} \frac{\partial n}{\partial \xi^{\prime}} \frac{\partial \xi^{\prime}}{\left(\xi-\xi^{\prime}\right)}+B n \frac{\partial n}{\partial \xi}+C \frac{\partial^{3} n}{\partial \xi^{3}}=0
$$

where $n \equiv n_{d}^{(1)}$, and the Landau damping, nonlinear and dispersion coefficients are

$$
\begin{aligned}
A & =\frac{\gamma_{1} M^{3}}{2}\left(\mu_{i 1} a_{2}+\mu_{i 2} b_{2}\right) ; \\
B & =\frac{\gamma_{2} M^{3}}{2}\left(\frac{3}{M^{2}}-\left(\mu_{i 1} a_{3}+\mu_{i 2} b_{3}\right) M^{2}\right) \\
C & =\frac{\gamma_{3} M^{3}}{2} .
\end{aligned}
$$

\section{Landau damping of DAWs}

To obtain the regular Landau damping of DAWs in plasmas, we set $B=C=0$. Then, Eq. (55) reduces to the following form:

$$
\frac{\partial n}{\partial \tau}+A \int_{-\infty}^{\infty} \frac{\partial n}{\partial \xi^{\prime}} \frac{\partial \xi^{\prime}}{\left(\xi-\xi^{\prime}\right)}=0
$$

Taking the Fourier transform of (59) and using that the inverse transform of $[$ isgn $(k)]$ is $-\frac{1}{\pi} P\left(\frac{1}{\xi}\right)$, we have

$$
\omega=-\iota \pi k \frac{\gamma_{1} M^{3}}{2}\left(\mu_{i 1} a_{2}+\mu_{i 2} b_{2}\right)
$$

Thus, due to the presence of $\delta=\sqrt{\frac{Z_{d} m_{i 1}}{m_{d}}}$, the dust acoustic waves suffer Landau damping due to inertial effects of colder ion population. The normalized Landau damping decrement is given as

$$
|\gamma|=\pi k \frac{\gamma_{1} M^{3}}{2}\left(\mu_{i 1} a_{2}+\mu_{i 2} b_{2}\right) \equiv \pi A
$$

To evaluate the importance of Landau damping, we have plotted the ratio of the Landau damping decrement to the phase velocity of linear DAWs in Fig 1. It is observed that as the nonthermalities of the cold and hot ions increase, the influence of Landau damping on the dynamics of the DAWs increases, i.e., for highly nonthermal plasma the Landau damping of DAWs due to colder ions becomes more significant. For lower values of the nonthermality 


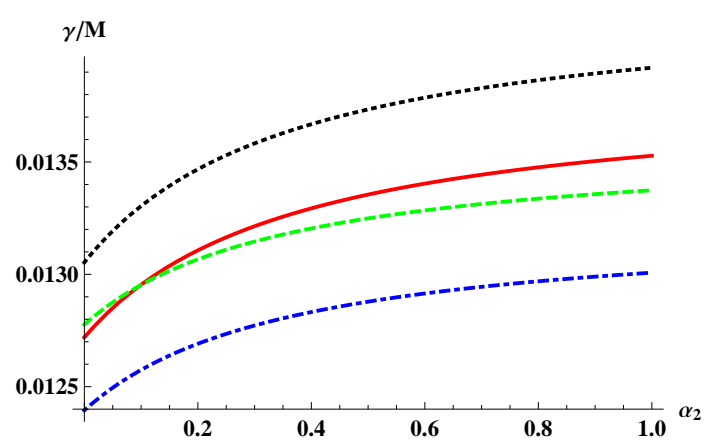

FIG. 1. (Color online) Variation of the ratio of the Landau damping decrement and the phase velocity of linear DAWs with nonthermality parameter for hot ions $\left(\alpha_{2}\right)$ for different values of the nonthermal parameter for the cold ions $\left(\alpha_{1}\right)$, the cold ions to dust density ratio $\left(\mu_{i 1}\right)$ and cold to hot ions temperature ratio $(\sigma)$. The parameters for red solid curve are given as $\alpha_{1}=0.2, \mu_{i 1}=0.7$, $\sigma=0.1, \gamma_{1}=0.01$. Blue (dotdashed) curve: $\mu_{i 1}=0.75$, black (dotted) curve: $\alpha_{1}=0.21$, green (dashed) curve: $\sigma=0.06$ with other parameters same as for the red curve.

of hot ions, the value of the Landau damping decrement is slightly higher for lower values of the cold to hot ion temperature ratio till a critical value of nonthermal parameter for the hot ions $\alpha_{2}$. Above this critical value of $\alpha_{2}$, there is an increase in ratio the of Landau damping to the linear phase velocity of the DAWs with an increase of the ion temperature ratio. In other words, as the temperature of the colder ions increase, the Landau damping has a more profound influence on the properties of linear DAWs. Also, with an increase in the number density of the colder ions, the effect of Landau damping on the DAWs becomes less dominant.

\section{ANALYTICAL SOLUTION OF THE KDV EQUATION WITH LANDAU DAMPING}

To obtain an analytical solution of Eq. (55), we follow the approach described in Refs. [2] and [13]. As $\gamma_{1} \rightarrow 0$, i.e., in the absence of Landau damping, Eq. (55) reduces to the usual $\mathrm{KdV}$ equation, with a solitary wave solution given by

$$
n=N \operatorname{sech}^{2}\left(\frac{\xi-U_{0} \tau}{W}\right)
$$

where $N=\frac{3 U_{0}}{B}$ is the amplitude, $W=(12 C / N B)^{1 / 2} \equiv \sqrt{4 C / U_{0}}$ is the width, and $U_{0}=$ $N B / 3$ is the constant phase speed (normalized by $C_{d}$ ). 
To emphasize certain properties of KdV equation with Landau damping, we integrate Eq. (55) with respect to $\xi$ and obtain ${ }^{2}$

$$
\frac{\partial}{\partial \tau} \int_{-\infty}^{+\infty} n d \xi=0
$$

i.e., Eq. (55) conserves the number of particles. Furthermore, multiplying Eq. (55) by n and integrating over $\xi$ yields

$$
\frac{\partial}{\partial \tau} \int_{-\infty}^{\infty} n^{2}(\xi, \tau) d \xi \leq 0
$$

where the equality sign holds only when $n=0$ for all $\xi$. Eq. (64) states that, an initial perturbation of the form (62) for which

$$
\int_{-\infty}^{+\infty} n^{2} d \xi<\infty
$$

will decay to zero. The peak amplitude $N$ of the wave is not a constant now but decreases slowly with time. Therefore, steady-state solutions such as shock waves do not exist with condition (65). However, steady-state shock solutions may exist for which the integral $\int_{-\infty}^{+\infty} n^{2} d \xi>\infty$, i.e., the integral of $n^{2}$ is divergent. We shall try to find the shock solution of the Landau damping modified KdV equation by numerically solving Eq. (55).

To determine the influence of a small amount of Landau damping on the solitary solution of famous $\mathrm{KdV}$ equation, we perform a perturbation analysis of Eq. (55) assuming that a $(\gg \epsilon)$ is a small parameter with $b, c \gg a$. A new space coordinate is introduced in a frame moving with group velocity of solitary wave and normalized to its width

$$
z=\left(\xi-\frac{B}{3} \int_{0}^{\tau} N d \tau\right) / W
$$

where $\mathrm{N}$ is assumed to vary slowly with time and $N=N(a, \tau)$. Also, assume that $n \equiv$ $n(z, \tau)$. Under this transformation, Eq. (55) becomes

$$
\frac{\partial n}{\partial \tau}+\frac{A}{W} P \int_{-\infty}^{+\infty} \frac{\partial n}{\partial z^{\prime}} \frac{d z^{\prime}}{z-z^{\prime}}-\left(\frac{N B}{3 W}-\frac{z}{2 N}\left(\frac{d N}{d \tau}\right)\right) \frac{\partial n}{\partial z}+\frac{B}{W} n \frac{\partial n}{\partial z}+\frac{C}{W^{3}} \frac{\partial^{3} n}{\partial z^{3}}
$$

where we have used $\partial n / \partial z^{6}=\partial n / \partial z$ at $z=z^{\prime}$. Next, to determine the solution of Eq. (67), we follow Refs. [2], [28] and [29] and generalize the multiple time scale analysis with respect to a. Thus, we consider the solution

$$
n(z, \tau)=n^{(0)}+a n^{(1)}+a^{2} n^{(2)}++a^{3} n^{(3)}+\ldots \ldots,
$$




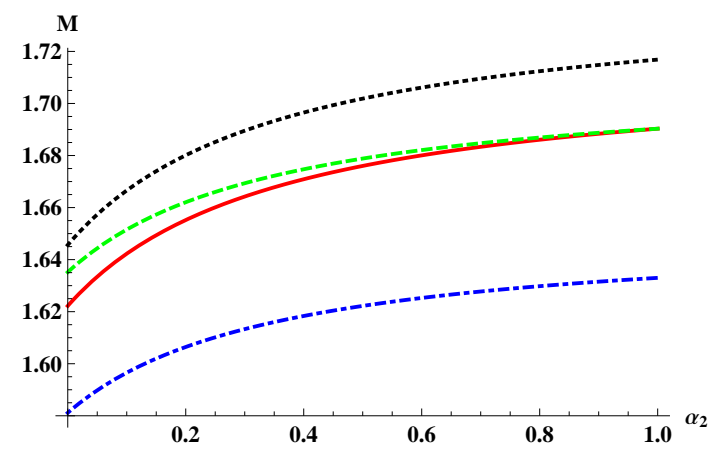

FIG. 2. (Color online) Variation of phase velocity $(M)$ with the nonthermality parameter for hot ions $\left(\alpha_{2}\right)$ for different values of the nonthermal parameter for the cold ions $\left(\alpha_{1}\right)$, the cold ions to dust density ratio $\left(\mu_{i 1}\right)$ and cold to hot ions temperature ratio $(\sigma)$. The parameters for red solid curve are given as $\alpha_{1}=0.2, \mu_{i 1}=0.7, \sigma=0.1$. Blue (dotdashed) curve: $\mu_{i 1}=0.75$, black (dotted) curve: $\alpha_{1}=0.21$, green (dashed) curve: $\sigma=0.08$ with other parameters same as for the red curve.

To obtain the analytical soliton solution of Eq. (55) with the Landau damping as

$$
n=N_{0}\left(1+\frac{\tau}{\tau_{0}}\right)^{-2} \operatorname{sech}^{2}\left[\left(\xi-\frac{B}{3} \int_{0}^{\tau} N d \tau\right) / W\right]+O(a)
$$

where $\tau_{0}$ is

$$
\tau_{0}=\frac{1.37}{A} \sqrt{\frac{3 C}{B N_{0}}}
$$

Eq. (69) shows that the addition of Landau damping in dynamics of dust acoustic (DA) solitary waves tends to algebraically decay the solitary amplitude with time. In order to understand the variation of solitary amplitude with various physical parameters, we perform a numerical investigation of Eq. (69) according to the data obtained by observations of Geotail satellite.

\section{NUMERICAL ANALYSIS}

Using the reductive perturbation method, we have derived a $\mathrm{KdV}$ equation including Landau damping for DA solitary waves propagating in an electron depleted dusty plasma containing two temperature nonthermal ions. In order to study the influence of various plasma parameters on the properties of DA solitary waves, we have performed a numerical investigation of various coefficients of Eq. (55) which is presented in Figs. 2-8. Dust charging typically occurs in a plasma due to the attachment of ions and electrons to the dust 


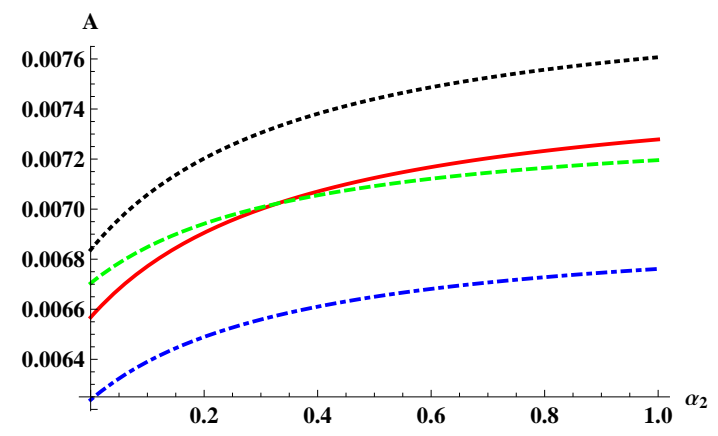

FIG. 3. (Color online) Variation of the Landau damping coefficient $(A)$ with nonthermality parameter for hot ions $\left(\alpha_{2}\right)$ for different values of nonthermal parameter for cold ions $\left(\alpha_{1}\right)$, the cold ions to dust density ratio $\left(\mu_{i 1}\right)$ and cold to hot ions temperature ratio $(\sigma)$. The parameters for red solid curve are given as $\alpha_{1}=0.2, \mu_{i 1}=0.7, \sigma=0.1, \gamma_{1}=0.01$. Blue (dotdashed) curve: $\mu_{i 1}=0.75$, black (dotted) curve: $\alpha_{1}=0.21$, green (dashed) curve: $\sigma=0.08$ with other parameters same as for the red curve.

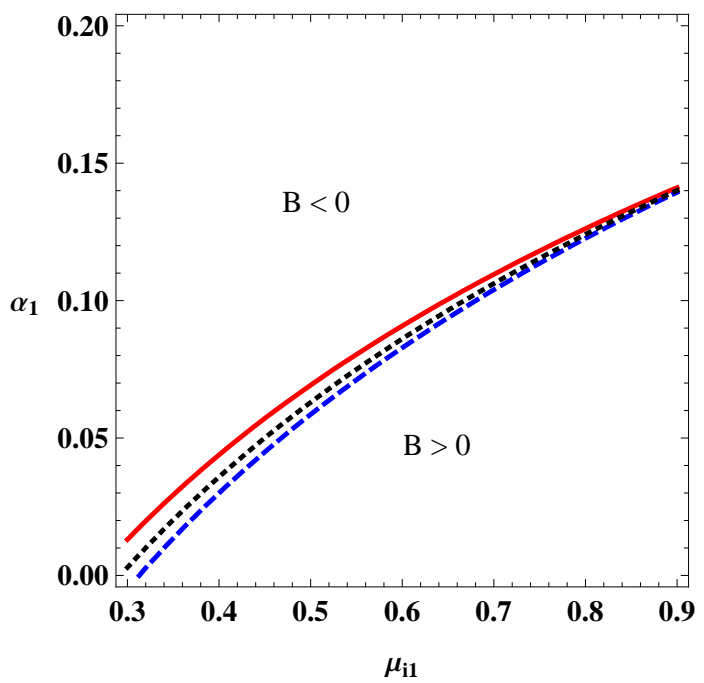

FIG. 4. (Color online) Contour plot for variation of the nonlinear coefficient $(B)$ with the nonthermality parameter for the cold ions $\left(\alpha_{1}\right)$ and the cold ion to dust density ratio $\left(\mu_{i 1}\right)$ for different values of the nonthermal parameter for hot ions $\left(\alpha_{2}\right)$ and the cold to hot ions temperature ratio $(\sigma)$. The parameters for the red solid curve are given as $\alpha_{2}=0.2, \sigma=0.1$, Blue (dashed) curve: $\alpha_{2}=0.5$, black (dotted) curve: $\sigma=0.08$ with $\gamma_{2}=0.5$.

grain $^{14,15}$. At equilibrium, the dust grains become negatively charged due to the higher flux of electrons to an uncharged surface. For numerical investigation, the parameters are taken to be $T_{i 1}=0.7 \mathrm{keV} ; T_{i 2}=7 \mathrm{keV}, m_{d}=4.6 \times 10^{-16} \mathrm{~kg}, Z_{d}=4.6 \times 10^{5}, m_{i 1}=2.65 \times 10^{-26} \mathrm{~kg}$ 


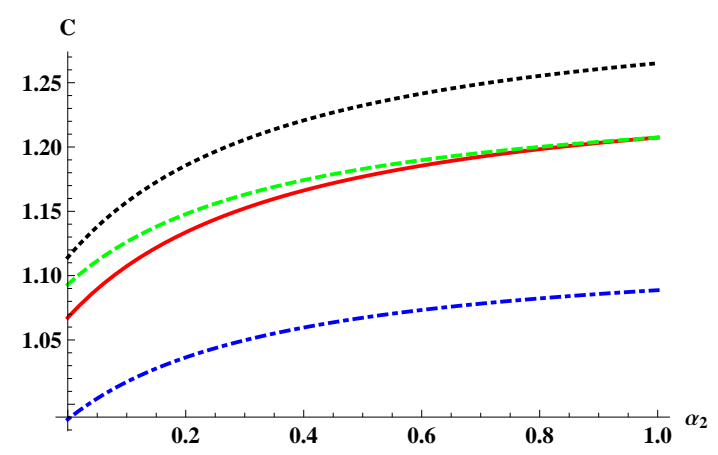

FIG. 5. (Color online) Variation of the dispersion coefficient $(C)$ with nonthermality parameter for hot ions $\left(\alpha_{2}\right)$ for different values of nonthermal parameter for cold ions $\left(\alpha_{1}\right)$, the cold ions to dust density ratio $\left(\mu_{i 1}\right)$ and cold to hot ions temperature ratio $(\sigma)$. The parameters for the red solid curve are given as $\alpha_{1}=0.2, \mu_{i 1}=0.7, \gamma_{3}=0.8, \sigma=0.1$. Blue (dotdashed) curve: $\mu_{i 1}=0.75$, black dotted curve: $\alpha_{1}=0.21$, green (dashed) curve: $\sigma=0.08$ with the other parameters same as for the red curve.

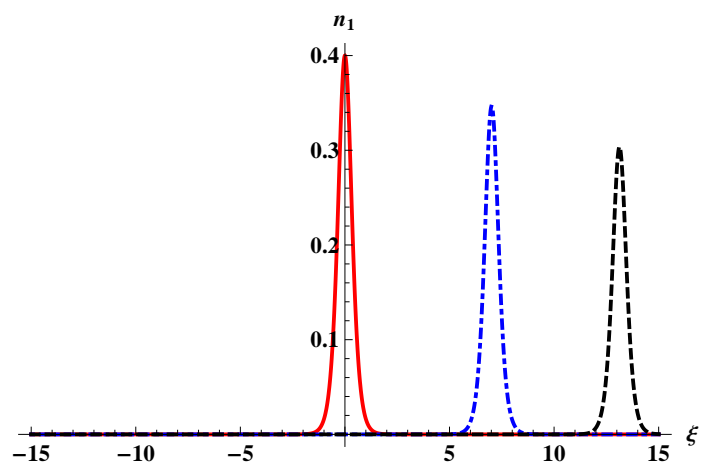

FIG. 6. (Color online) Time evolution of the solitary wave profile under the influence of Landau damping. Red (solid) curve: $\tau=0$; Blue (dotdashed) curve: $\tau=50$; Black (dashed) curve: $\tau=100$. The values of the physical parameters being $\mu_{i 1}=0.7, \alpha_{1}=0.2, \alpha_{2}=0.8, \gamma_{1}=0.01$, $\gamma_{2}=0.5, \gamma_{3}=0.8, \sigma=0.1$

for the $\mathrm{O}^{+}$ions and $m_{i 2}=1.672 \times 10^{-27} \mathrm{~kg}$ for the $H^{+}$ions. Fig. 2 depicts variation of the phase velocity $M$ with nonthermality of cold and hot ions (via $\alpha_{1}, \alpha_{2}$ ), cold ions to dust density ratio $\left(\mu_{i 1}\right)$ and cold to hot ions temperature ratio $(\sigma)$. The phase velocity of the DA solitary waves increases with increasing nonthermality of the hot and cold ions. In other words, the DA solitary waves propagate faster if there are more nonthermal ions present. It is also observed that the phase velocity of DA solitary waves decreases with increase in cold ions to dust density ratio $\left(\mu_{i 1}\right)$ as well as with an increase in the ion temperature ratio $(\sigma)$. 


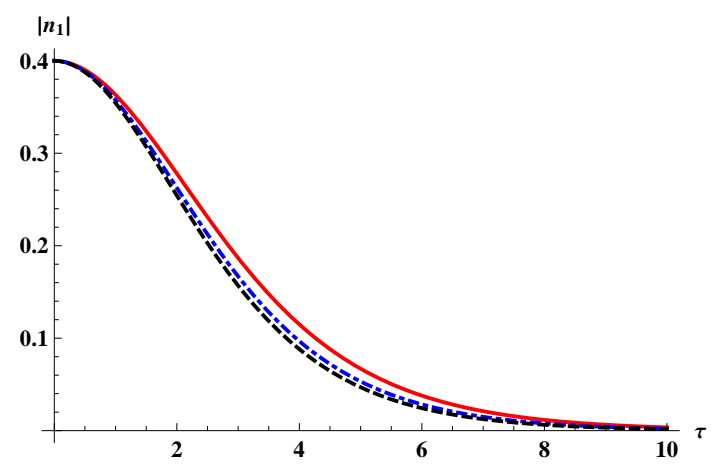

FIG. 7. (Color online) The variation of the peak amplitude of a soliton with time under the influence of Landau damping for different values of the nonthermal parameter for cold ions $\left(\alpha_{1}\right)$. Red (solid) curve: $\alpha_{1}=0.2$, blue (dotdashed) curve: $\alpha_{1}=0.3$, black (dashed) curve: $\alpha_{1}=0.4$. Other parameters are $N_{0}=0.4, U_{0}=40, \mu_{i 1}=0.7, \alpha_{2}=0.8, \gamma_{1}=0.01, \gamma_{2}=0.5, \gamma_{3}=0.8$, $\sigma=0.1$.

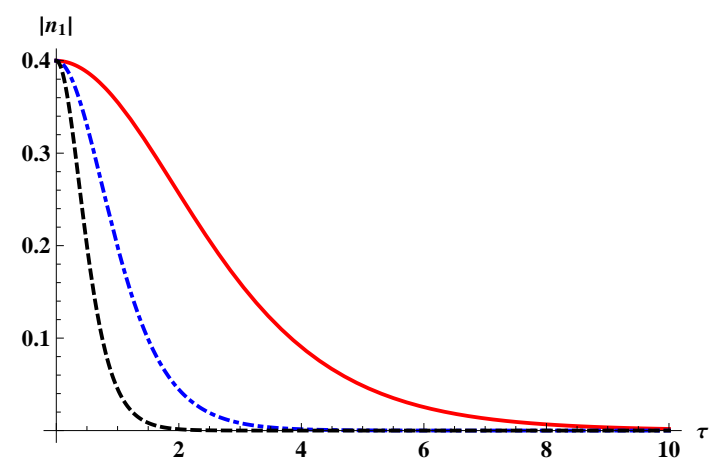

FIG. 8. (Color online) The variation of the peak amplitude of a soliton with time under the influence of Landau damping for different values of the nonthermal parameter for hot ions $\left(\alpha_{2}\right)$. Red (solid) curve: $\alpha_{2}=0.3$, blue (dotdashed) curve: $\alpha_{2}=0.6$, black (dashed) curve: $\alpha_{1}=0.9$. Other parameters are $N_{0}=0.4, U_{0}=40, \mu_{i 1}=0.7, \alpha_{1}=0.2, \gamma_{1}=0.01, \gamma_{2}=0.5, \gamma_{3}=0.8$, $\sigma=0.1$.

This may be due to that with an increase in the temperature of the hot ions or as the hot ions become more nonthermal, the streaming of ions through the layers of plasma becomes fast. However, for highly nonthermal ions, the phase velocity of DA solitary waves does not show much variation with the cold to hot ions temperature ratio.

Fig. 3 depicts the variation of the Landau damping coefficient $(A)$ with different physical parameters. It is to be noted here that the magnitude of Landau damping is enhanced as both the ion species become more nonthermal similar to the case of variation of phase 


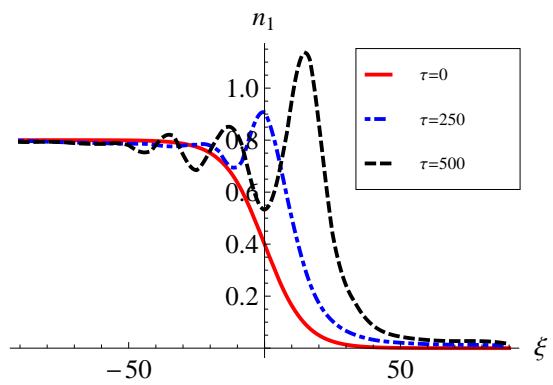

FIG. 9. (Color online) Time varying numerical solution of KdV equation with Landau damping. The parameters being $N_{0}=0.4, \alpha_{1}=0.15, \alpha_{2}=0.8, \mu_{i 1}=0.7, U_{0}=40, \gamma_{1}=0.01, \gamma_{2}=0.5$, $\gamma_{3}=0.8, \sigma=0.1$.

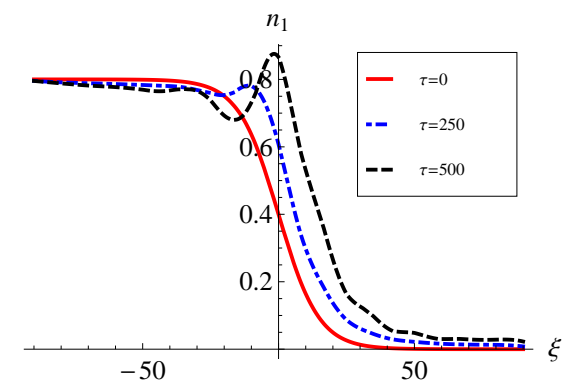

FIG. 10. (Color online) Time varying numerical solution of KdV equation with Landau damping. The parameters being $N_{0}=0.4, \alpha_{1}=0.25, \alpha_{2}=0.8, \mu_{i 1}=0.7, U_{0}=40, \gamma_{1}=0.01, \gamma_{2}=0.5$, $\gamma_{3}=0.8, \sigma=0.1$.

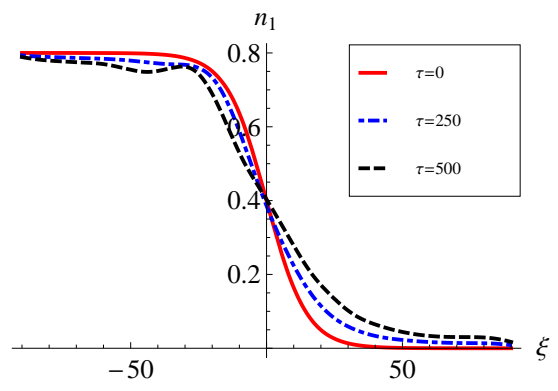

FIG. 11. (Color online) Time varying numerical solution of the KdV equation with Landau damping. The parameters being $N_{0}=0.4, \alpha_{1}=0.35, \alpha_{2}=0.8, \mu_{i 1}=0.7, U_{0}=40, \gamma_{1}=0.01, \gamma_{2}=0.5$, $\gamma_{3}=0.8, \sigma=0.1$. 
velocity $(M)$ with different physical parameters. The physical interpretation is that with an increase in the nonthermal parameter for the ions, the number of energetic ions in the tail of the energy spectrum increases and a larger number of particles extract energy from the wave and the Landau damping increases. It is also inferred that as the cold ions to dust density ratio increases, i.e., the dust concentration decreases, the magnitude of the Landau damping coefficient decreases. In other words, a decrease in the concentration of inertial dust particles tends to decrease the rate of Landau damping of the DAWs. With an increase in cold to hot ions temperature ratio, the Landau damping coefficient decreases (increases) for lower (higher) values of the nonthermal parameter for the hot ions. To illustrate the existence region of parameters for different polarity solitary structures, a contour plot that represents variation of the nonlinear coefficient $(B)$ with the nonthermal parameter of the cold ions $\left(\alpha_{1}\right)$ and the cold ions to dust density ratio $\left(\mu_{i 1}\right)$ for different values of the nonthermal parameter for hot ions $\left(\alpha_{2}\right)$ as well as the cold to hot ions temperature ratio $(\sigma)$ is shown in Fig. 4. The physical parameters regime above the curves represents the existence of rarefactive nonlinear structures, whereas the parametric regime below the curves represent compressive nonlinear structures. It can be easily inferred from the graphs that the critical number density ratio of cold ions to dust concentration $\mu_{i 1}$ (at which transition from compressive to rarefactive nonlinear structures occurs) has a higher value if the nonthermality of the cold and hot ions is increased. However, the critical number density ratio decreases with an increase in the cold to hot ion temperature ratio $(\sigma)$. The variation of the dispersion coefficient of the KdV equation with Landau damping with various physical parameters is presented in Fig. 5. The value of the dispersion coefficient shows a similar trend as that of the Landau damping coefficient with a variation of the associated physical parameters. However, the value of dispersive term is several orders of magnitude higher than that of Landau damping coefficient. The analytical solution of the KdV equation with Landau damping (Eq. (55)) as obtained in Eq. (69) is illustrated in Fig. 6. It is a well known fact that the balance of nonlinearity and dispersion in a plasma can lead to the formation of stable localized structures called solitons. In the case when $A \rightarrow 0$, Eq. (55) reduces to the well known KdV equation which has solutions in the form of solitons. However, the presence of an additional Landau damping term tends to decay the amplitude of the solitary wave with time, which is evident from Fig. 6 which 
depicts the time evolution of a soliton under the influence of Landau damping. The variation of the peak amplitude of the solitary structures with the nonthermality of cold ions is presented in Fig. 7. It is seen that the decay of the peak solitary amplitude is faster as the cold ions are more nonthermal. Similar is the case of varying the nonthermality of the hot ions as illustrated in Fig. 8, i.e., the decay in the peak amplitude of the soliton becomes more rapid with an increase in the nonthermality of hot ions and the variation is substantial as compared to the case of increasing nonthermality of the colder ions.

\begin{tabular}{|c|c|c|c|}
\hline$\alpha_{1}$ & $\mathrm{~A}$ & $\mathrm{~B}$ & $\mathrm{C}$ \\
\hline 0.08 & 0.00423 & 0.142442 & 1.06 \\
0.09 & 0.00443 & 0.07935 & 1.120 \\
0.1 & 0.0046 & 0.010147 & 1.179 \\
\hline
\end{tabular}

TABLE I. The values of the nonlinear, dispersion and Landau damping coefficients used to evaluate the numerical solution for different values of the nonthermal parameter for the cold ions. Other parameters are $\mu_{i 1}=0.7, \gamma_{1}=0.01, \gamma_{2}=0.5, \gamma_{3}=0.8, \sigma=0.1, \alpha_{2}=0.8$.

The balance of nonlinearity and dissipation (due to Landau damping) gives rise to shocklike structures in plasma. Shock waves are characterized as an abrupt jump in the plasma potential, density etc. To illustrate the influence of varying the nonthermality of the ions on the Landau damping modified shock-like solution, we shall use the values of various coefficients of Landau damping modified KdV equation (55) for different values of the nonthermality of the cold ions as given in Table 1. In order to follow the time evolution of the shock-like solution of the KdV equation with Landau damping, we use a MATHEMATICA based finite difference scheme to numerically solve Eq. (55). To obtain the time varying numerical solution, we shall use a shock-like pulse as the initial waveform as

$$
n(0, \xi)=A_{\text {ini }}\left(1-\tanh \left(k_{0} \xi\right)\right), \xi \in(-L, L)
$$

where, $L$ depicts the spatial length and $A_{i n i}$ the shock amplitude. The boundary conditions are $n(\tau,-L)=A_{\text {ini }}\left(1+\tanh \left(k_{0} L\right)\right)$ and $\phi^{(1)}(\tau, L)=0$. For numerical analysis, we take $L=100$ and simulate Eq. (55) using the values of the coefficients given in Table I. The Landau damping term is numerically integrated using a trapezoidal rule. The time varying solutions of the KdV equation with Landau damping for values of the nonthermal parameter for the cold ions as $\alpha_{1}=0.08, \alpha_{1}=0.09$ and $\alpha_{1}=0.1$ are shown in Figs. 9, 10 and 11, 
respectively. It is inferred from the Fig 9 that if the colder ions are less nonthermal (i.e., more Maxwellian), the initial shock-like compression pulse propagates with increasing amplitude and develops an oscillatory tail, i.e., as time elapses, the dispersive effects begin to dominate the plasma dynamics and a monotonic shock transits into an oscillatory shock profile due to the balance of dissipative, nonlinear and dispersive effects. The time evolution of the shock profile for an increased nonthermality of the cold ions is presented in Fig 10. It is observed that as the value of nonthermal parameter increases (at $\alpha_{1}=0.09$ ), the dispersive effects become less dominant and the monotonic character of the shock wave is retained for a longer duration in time. In other words, we can say that the monotonic shock structures are found to be temporally more stable as the colder ion species becomes more nonthermal. As we further increase the value of the nonthermal parameter for the cold ions $\alpha_{1}=0.1$, we observe that the nonlinearity in the system is unable to balance the dissipation due to Landau damping and the monotonic shock structure decays with time (see Fig. 11). As it is also evident from Table I that the value of the Landau damping coefficient $A$ increases, whereas that of the nonlinear coefficient $B$ decreases with increase in the nonthermality of cold ions. It is, however noteworthy that as the colder ions approach a Maxwellian distribution, the dispersive effects dominate and the monotonic shock structure transits into an oscillatory shock. The nonthermality of the hot ions does not incur a profound influence on the evolution of the shock structures in the plasma. It is remarked that the solitary wave experiences a larger Landau damping rate for a high concentration of nonthermal hot ions as compared to cold ions and the peak amplitude decays due to the dominance of increasing dissipative effects arising through Landau damping with the increase in the nonthermality of ions. Similar effects are observed for an initial shock-like pulse, where an oscillatory shock structure at later times tends to retain a monotonic character due to a subtle balance between nonlinear and dissipative effects with an increase in the nonthermality of cold ions. Hence, the relatively weak dissipative effects due to Landau damping even being lower in magnitude may be significant for the evolution of nonlinear structures in a dusty plasma. 


\section{CONCLUSIONS}

We have investigated the influence of Landau damping and nonthermality of the ions on the propagation properties of dust acoustic (DA) solitary and shock waves in a dusty plasma comprising two temperature ions motivated by the observations of the Geotail spacecraft. An analytical solution of the $\mathrm{KdV}$ equation with an additional Landau damping term is investigated. It is found that the solitary amplitude decays with time due to the presence of an additional Landau damping term in the $\mathrm{KdV}$ equation. The decrease of the peak amplitude becomes more rapid with increase in the nonthermality of ions, whereas the decay is observed to be faster for highly nonthermal hot ions as compared to more nonthermal cold ions. On the other hand, under certain conditions when the nonlinearity is balanced by dissipation, shock-like structures are formed. The time evolution of the shock waves is investigated as a numerical solution of the modified KdV equation including Landau damping. It is seen that an initially compressional shock-like pulse forms a monotonic shock profile for a higher nonthermality of the cold ions, whereas oscillatory shock structure is formed as the nonthermality of cold ions is decreased, i.e., as the cold ions approach Maxwellian distribution. Motivated by the reports of the Geotail spacecraft and observation of highly energetic charged particles in space, we have for the first time investigated the influence of Landau damping due to cold ions on dust acoustic nonlinear structures (shocks and solitons) in the presence of two temperature nonthermal ions. It is noteworthy that the Landau damping of DA waves due to cold $\mathrm{O}^{+}$ions incurs a profound influence on the propagation characteristics of DA nonlinear structures and hence, the inertia of heavier $\mathrm{O}^{+}$ions shall not be neglected while studying the dynamics of nonlinear waves in this particular region of space. The findings of present investigation shall be very useful in understanding the physics of resonant wave-particle interactions in an electron depleted dusty plasma environment where two temperature electrons have been observed such as Earth's magnetosphere and Saturn's F-ring.

\section{ACKNOWLEDGMENT}

This work by N.S.S. was supported by DRS-II (SAP) No. F/530/17/ DRS-II/2015(SAPI) University Grants Commission (UGC), New Delhi, India. Y.G. gratefully acknowledges 
University Grants Commission (UGC) for providing scholarship under Basic Scientific Research (BSR) scheme.

1 F.F. Chen, Introduction to Plasma Physics and Controlled Fusion, (Springer, Berlin 2006).

2 E. Ott and R. Sudan, Phys. Fluids, 12, 2388 (1969).

3 A. Hirose, I. Alexeff, W.D. Jones, N.A., Krall, D. Montgomery, Phys. Lett. A 29, 31 (1969).

4 V.I. Karpman, J.P., Lynov, P. Michelsen, H.L. Pecseli, J.J. Rasmussen, Phys. Fluids 23, 1782 (1980).

5 A. Barman and A.P. Misra, Phys. Plasmas, 21, 073708 (2017).

6 A. Sikdar and M. Khan, J. Theor. Appl. Phys., 11, 137 (2017).

7 Y. Saitou and Y. Nakamura, Phys. Plasmas, 10, 11 (2003).

8 Y. Nakamura, H. Bailung and Y. Saitou, Phys. Plasmas, 11, 3925 (2004).

9 A. Bandyopadhyay and K. P. Das, Phys. Plasmas, 9, 465 (2002).

10 A. Bandyopadhyay and K.P. Das, Phys. Plasmas, 9, 3333 (2002).

11 S. Ghosh and R. Bharuthram, Astrophys. Space Sci., 331, 163 (2011).

12 H. Kozima, K. Yamagiwa, T. Tanaka, H. Matsubara, H. Yoshino, J. Physical Soc. Japan, 58, 504-510 (1989).

13 Y. Saitou, Y. Nakamura, Phys. Plasmas, 10, 11 (2003).

14 J. Goree, Plasma Sources Sci. Technol., 3, 400-406 (1994).

15 P.K. Shukla and B. Eliasson, Rev. Mod. Phys. 81, 25-44 (2009).

16 P. K. Shukla and A. A. Mamun, Introduction to Dusty Plasma Physics (Institute of Physics Publishing, Bristol, 2002), pp. 1-35.

17 N.N. Rao, P.K. Shukla and M.Y. Yu, Planet. Space Sci. 38, 543-546 (1990).

18 P.K. Shukla, V.P. Silin, Phys. Scripta. 45, 508 (1992).

19 Y. Ghai and N.S. Saini, Astrophys. and Space Sci., 58, 362 (2017).

20 M. Ferdousi, M.R. Miah, S. Sultana, A.A. Mamun, Astrophys. Space Sci. 360, 43 (2015).

21 R. Blandford, D. Eichler, Phys. Reports 154, 1-75 (1987).

22 N. Shimada, T. Terasawa, J.K. Jokipii, J. Geophys. Res. 102, 22301-22310 (1997).

23 M. Shahmansouri and A. A. Mamun: Phys. Plasmas, 80, 593 (2014).

24 B.S. Chahal, Y. Ghai, N.S. Saini, J. Theor. and Appl. Phys., 11, 181189 (2017). 
25 U. K. Samanta, A. Saha and P. Chatterjee, Phys. Plasmas, 20, 022111 (2013).

26 A. Saha and P. Chatterjee, Eur. Phys. J. D, 69, 203 (2015).

27 R.A. Cairns, A. A. Mamun, R. Bingham, R. Boström, R.O. Dendy, C.M.C. Nairn and P.K. Shukla, Geophys. Res. Lett., 22, 2709 (1995).

28 R.A. Cairns, A.A. Mamun, R. Bingham and P.K. Shukla, Phys. Scripta T63, 80 (1996).

29 P.O. Dovner, A.I. Eriksson, R. Bostrom, and B. Holback, Geophys. Res. Lett., 21, 18271830 (1994).

30 M.N. Khattak, A. Mushtaq, A. Qamar: Braz. J. Phys., 45, 633 (2015).

31 I. Tasnim, M.M. Masud, M.G.M. Anowar, and A.A. Mamun: IEEE Trans. Plasma Sci., 43, 7 (2015).

32 S. Dalui, A. Bandyopadhyay and K.P. Das: Phys. Plasmas, 24, 042305 (2017).

33 F. Verheest and M.A. Hellberg: Phys. Plasmas, 24, 022306 (2017).

34 K. Seki, M. Hirahara, M. Hoshino, T. Terasawa, R.C. El Phic, Y.T. Saito Mukai, H. Hayakawa, H. Kojima, M. Matsumoto: Nature,422, 589 (2003).

35 M.N. Nishino, T. Terasawa, M. Hoshino, COSPAR Colloq. Ser. 16, 28 (2005).

36 M. Horanyi, L.F. Houpis and D. A. Mendis, Astrophys. Space Sci, 144, 215-229 (1988).

37 S. I. Popel and T. I. Morozova, Plasma Phys. Reports, 43, 566-575 (2017). 


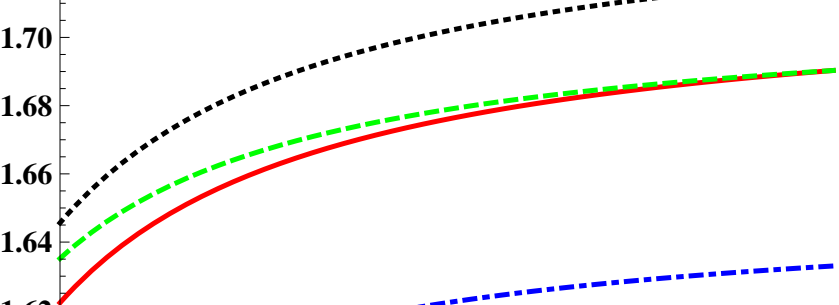

1.62

1.60 


\subsection{6}

0.0074

0.0072

0.0066

0.0070

0.0064

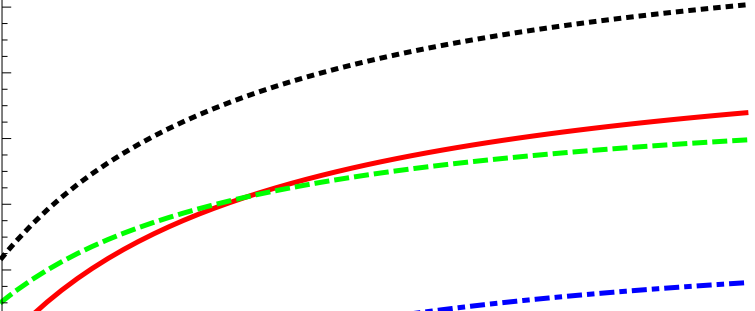




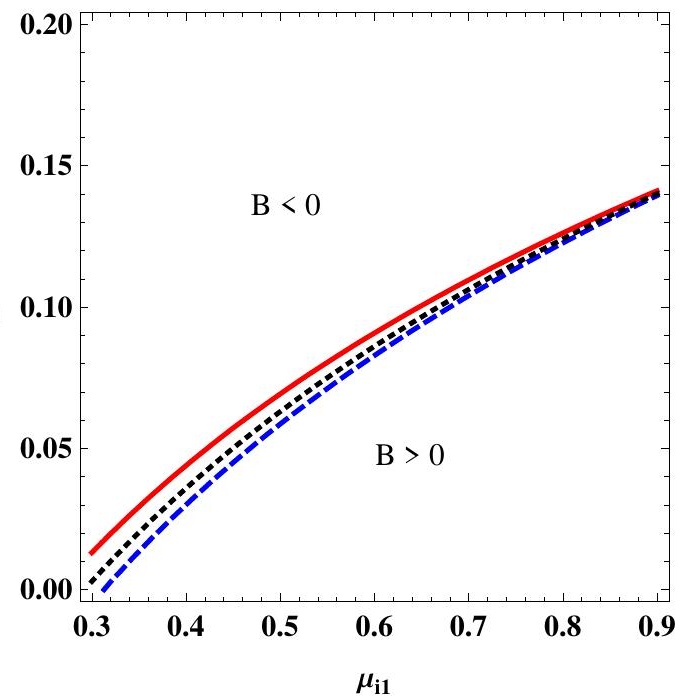




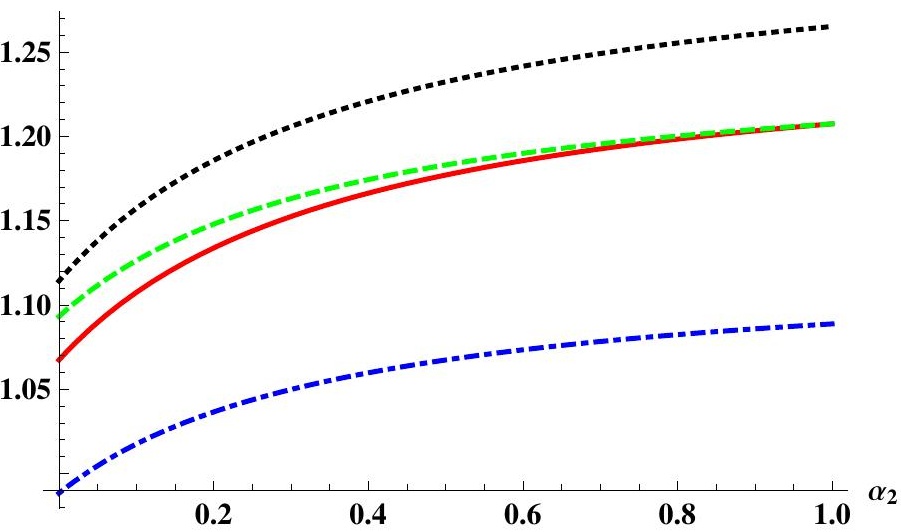


$\left|n_{1}\right|$

0.4

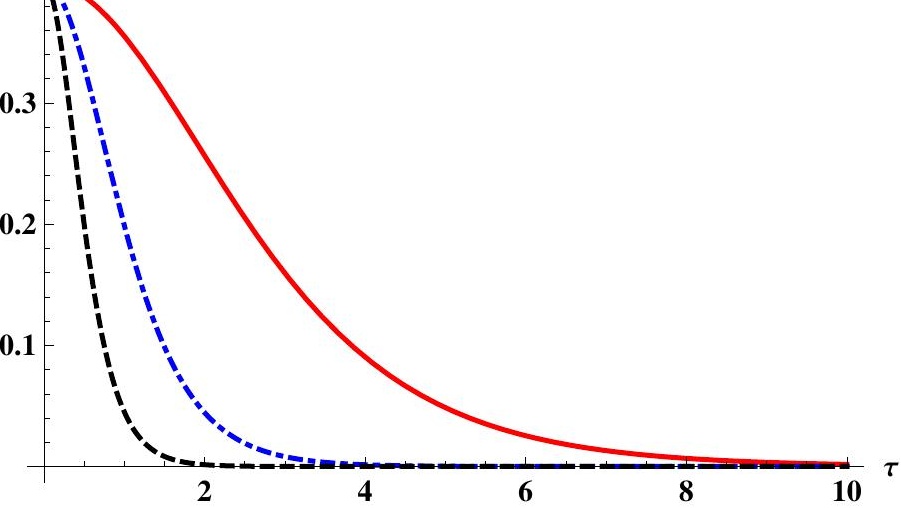




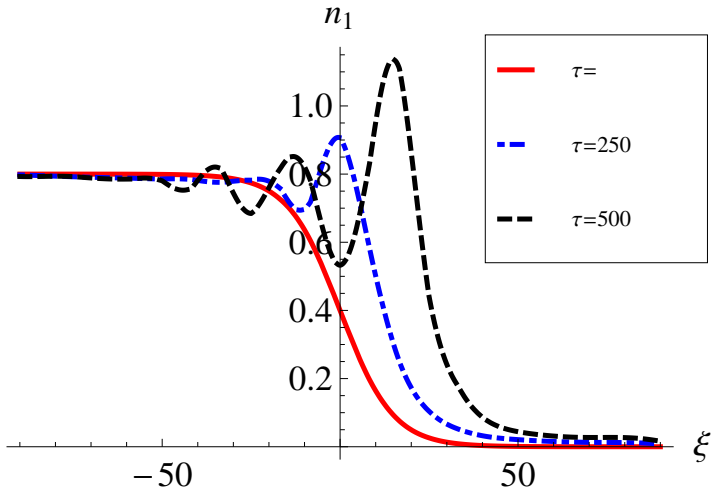




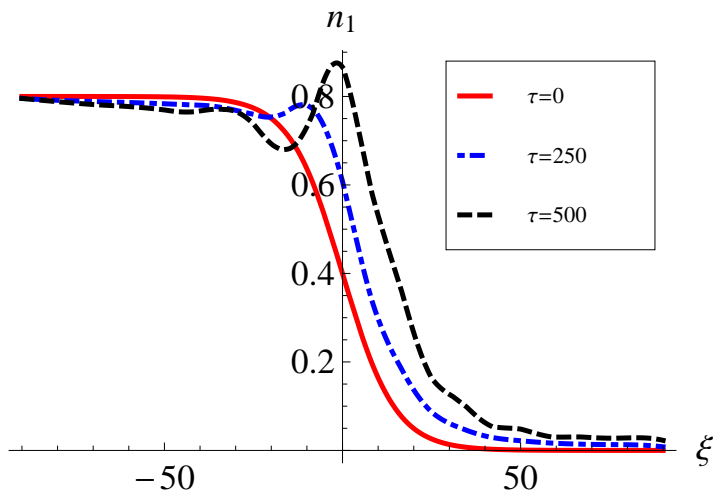




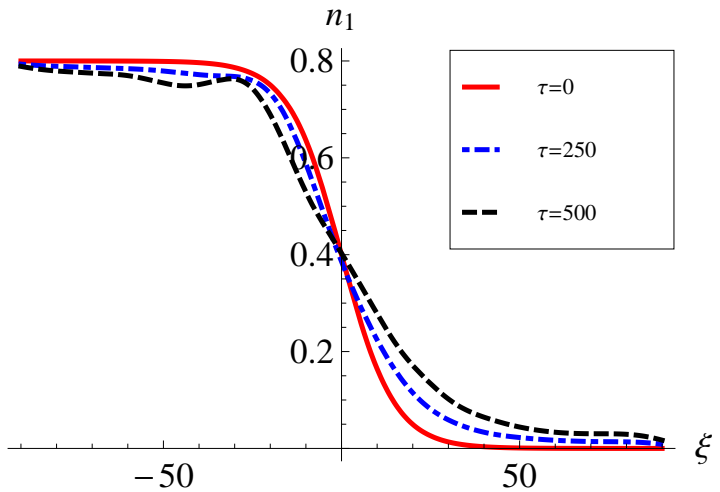

